\title{
Aproximación epistemológica a la investigación en comunicación: significados de comunicación, disciplinariedad y criterios para construir una disciplina
}

\section{Epistemological approach to communication research: meanings of communication, disciplinarity and criteria for building a discipline}

Gloria Gómez-Diago. Universidad Rey Juan Carlos. Madrid, España. gloria.gomez.diago@urjc.es

$[\underline{\mathrm{CV}}]$ (1) $\mathrm{C}$

Cómo citar este artículo / Referencia normalizada

Gómez-Diago, G. (2020). Aproximación epistemológica a la investigación en comunicación: significados de comunicación, disciplinariedad y criterios para construir una disciplina. Revista Latina de Comunicación Social, (77), 393-412. https://www.doi.org/10.4185/RLCS-2020-1464

\begin{abstract}
RESUMEN
Introducción: En "Ferment in the Field" (1983), hace 37 años, Katz planteaba que lo mejor que le había sucedido a la investigación en comunicación era dejar de buscar evidencias de la capacidad de los medios para cambiar opiniones, actitudes y acciones a corto plazo; para analizar su función en la configuración de nuestras imágenes de la realidad. Mattelart (1983) animaba a estudiar la interacción entre audiencias y medios desde una perspectiva no comercial y Ewen (1983) proponía utilizar historias orales o fuentes literarias. Cuatro décadas después, siguen estudiándose los efectos a corto plazo de los medios, predominando el análisis de sus contenidos (Martínez Nicolás y Saperas, 2011, 2016), análisis este, el de los contenidos de los medios, sobre el que, como sucedía hace treinta años (Cáceres y Caffarel, 1992; p. 12), el campo parece sustentar su especificidad, adoleciendo de una institucionalización intelectual (Peters, 1986; Lacasa, 2017) que puede ser colmada mediante una metainvestigación de las ideas que destile perspectivas, conceptos y métodos que se han venido utilizando en la investigación en comunicación. Método: Tras analizar tres volúmenes referentes en metainvestigación, los volúmenes del Journal of Communication "Ferment in the Field" (1983) y "The Future of the Field. Between fragmentation and cohesion" (1993) y el volumen 1 de Rethinking Communication (1989), "Paradigm Issues". Resultados: Se aportan perspectivas respecto de maneras de entender la comunicación, respecto del carácter disciplinar del campo y en lo que se refiere a los requisitos necesarios para convertir el campo en disciplina. Las perspectivas y propuestas emergen, principalmente, de dos maneras de entender la comunicación: como producto o resultado y como relación.
\end{abstract}


PALABRAS CLAVE: epistemología; Ferment in the field; The Future of the Field. Between fragmentation and cohesion; historia de la investigación en comunicación; metainvestigación en comunicación; métodos de investigación; Paradigm Issues; teoría de la comunicación.

\begin{abstract}
Introduction: In "Ferment in the Field" (1983), 37 years ago, Katz stated that the best thing that had happened to communication research was to stop looking for evidence of the media's ability to change opinions, attitudes and actions in the short term to analyze its role in the configuration of our images of reality. Mattelart (1983) encouraged to study the interaction between audience and media from a non commercial perspective and Ewen (1983) proposed using oral histories or literary sources. Four decades later, the short-term effects of media continue to be studied and predominates the analysis of their content (Martínez Nicolás and Saperas, 2011, 2016), analysis, that of media contents, on which as it happened thirty years ago (Cáceres and Caffarel, 1992; p. 12) the field seems to support its specificity, suffering the lack of an intellectual institutionalization (Peters, 1986; Lacasa, 2017) which can be filled through a meta-research of ideas, by distilling perspectives, concepts and methods that have been used in communication research. Method: Through the analysis of three reference volumes in meta-research, the volumes of the Journal of Communication "Ferment in the Field" (1983) and "The Future of the Field. Between fragmentation and cohesion" (1993) and volume 1 of Rethinking Communication (1989) "Paradigm Issues". Results: We will be bringing perspectives regarding the meanings of communication, the disciplinary character of the field and regarding the requirements needed for turning the field into a science. The perspectives and proposals emerge, mainly, from two ways of understanding communication: as product or result and as a relationship.
\end{abstract}

KEYWORDS: epistemology; Ferment in the field; The Future of the Field. Between fragmentation and cohesion; history of communication research; metaresearch in communication; methodology; communication theories; communication theory; Paradigm Issues; research methods.

\title{
CONTENIDOS
}

1. Introducción. 2. Objetivos. 3. Método de investigación y muestra. 4. Resultados 4.1. Significados de la comunicación. 4.2. Criterios/procedimientos para convertir el campo de la comunicación en una disciplina. 5. Discusión y Conclusiones. 6. Referencias.

\section{Introducción}

Estamos en un contexto en el que en la práctica de la Ciencia Social es posible utilizar entornos y herramientas informáticas que proporcionan diversidad y cantidad de datos, en un contexto en el que en ocasiones se obvia situar la importancia y la utilidad de dichos datos, produciendo un tipo de literatura científica que ya puede ser generada por robots (Gunkel, 2015). Además, en la investigación social y específicamente en la investigación en comunicación es cada vez más frecuente el uso de plataformas como Qualtrics o Mechanical Turk. Estas plataformas pretenden acercar a los investigadores a unos actores sociales que, al otro lado de la pantalla, responden a preguntas normalmente cerradas y a estímulos en experimentos que suelen reducirse a demostrar un fenómeno, perdiendo pues su función investigadora, como ocurre con los experimentos que se orientan a la demostración, en lugar de funcionar como "generadores de sorpresa" (Hoagland, 1990) o como "máquinas construir el futuro" (Jacob, 1987), en Rheinberger (2011, p. 312).

Mientras la investigación social utiliza cada vez más formulas para hacer un acercamiento a la sociedad que guarda las distancias (la física, la intelectual y la emocional) con los actores sociales, 
dificultando así la que Mills (1987, p. 32) considera como la primera tarea política e intelectual del científico social, esto es "poner en claro los elementos del malestar y la indiferencia contemporáneos"; las tasas de desempleo ${ }^{1}$ y de desigualdad siguen aumentando, habiéndose visto multiplicadas por la Covid-19.

La investigación en comunicación puede ayudar en diferentes niveles a la sociedad, a la humanidad, de igual manera que lo hacen otras disciplinas; pero parece necesario revisar críticamente las implicaciones sociales de nuestro discurso y revisar nuestro vocabulario (Krippendorff, 2017, p. 98), parece necesario redefinir y/o ampliar el significado de la investigación en comunicación y abrir su foco, sus métodos y su ámbito de actuación.

La metainvestigación es una línea de trabajo que puede tener diversas finalidades y que se utiliza en diferentes disciplinas, siendo común en la Sociología, la Filosofía, la Psicología, la Ciencia política o la Historia (Ritzer, 2009, p. 5). Danziger, en el campo de la Psicología, ha venido realizando una metainvestigación orientada a identificar las perspectivas, las ideas y los conceptos sobre los que se ha ido generando ese campo científico. El investigador (2003, p. 23) subraya la importancia de los "objetos psicológicos", es decir, de los conceptos que han venido dando forma al campo de la psicología y destaca la necesidad de identificar las maneras en las que estos objetos se utilizan en la práctica científica y las formas en las que esos conceptos han ido evolucionando, si es el caso, como sucede, según Danziger con el concepto de "behaviour". Este tipo de análisis es lo que el investigador llama "biografía de objetos científicos". Los conceptos y las maneras en las que éstos se entienden son también fundamentales en el campo de la investigación en comunicación (GómezDiago, 2017). En este sentido, Corner (1979) planteaba la necesidad de seguir utilizando el concepto de "comunicación de masas", Hackett (1984) señala el error de utilizar en la investigación en comunicación conceptos como el de "objetividad", Newcomb (1986) alertaba ya hace tres décadas de que los conceptos de "emisor", "mensaje" o "receptores", apenas permitían rasgar en la superficie de los cambios experimentados en la sociedad y Krippendorff (2017) propone abandonar los conceptos de "contenido del mensaje", "poder" y "framing". El investigador expresa que si bien hay conceptos que pueden ser populares en la conversación cotidiana sobre comunicación, usarlos sin pensar en nuestro discurso académico limita la capacidad de reconocer lo que hacen, imponiendo involuntariamente restricciones a las preguntas de investigación, y reproduciendo o sirviendo a instituciones sociales potencialmente opresivas. En esta línea que considera a los conceptos y a las formas de entenderlos como definitorios de la investigación en comunicación que se realiza, Hall (1989, p. 47) expresa que la transición del paradigma dominante al paradigma crítico tiene que ver con maneras opuestas de entender los elementos de la práctica comunicativa. El investigador plantea que la concepción de que un contenido particular puede ser aislado en términos de conducta y de que se pueden medir sus efectos, tiene que ser reemplazada por una perspectiva que entienda los aspectos culturales de cada elemento, así como su carácter semiótico y discursivo, una perspectiva que reconozca que los medios funcionan en y a través del significado y que entienda que no hay un "mensaje" que está ya en la realidad, un mensaje que el lenguaje u otros medios lleva a una mentes y a unas conciencias vacías, pues el significado es polisémico y está muy vinculado al contexto.

\footnotetext{
${ }^{1}$ España es, de acuerdo con Eurostat (2019) el segundo país de la Unión Europea con más desempleo (14,1 \%), siendo Grecia el primero (17,3\%). Esta tasa de desempleo es menor de la real dado que para su cálculo se tiene en cuenta la definición recomendada por la Organización Internacional del Trabajo, que reconoce como desempleados a personas entre 15 y 74 años de edad que están sin trabajo, que están disponibles para comenzar a trabajar dentro de las próximas dos semanas y que han buscado empleo activamente en algún momento durante las cuatro semanas anteriores. Fuera de la definición de "desempleado" quedan pues personas como aquellas que, con cincuenta años o más, dejen de buscar trabajo durante un mes o más tiempo y también aquellas que se han rendido por la dificultad añadida, en muchas ocasiones, de ser remplazados por jóvenes que trabajan como becarios sin recibir salario alguno, aún después de haberse graduado.
} 
En un contexto en el que se demanda la necesidad de una institucionalización intelectual para el campo de la comunicación (Peters, 1986; Lacasa 2017), parece fundamental indagar en qué significa, en qué se puede entender por comunicación. Saber qué entendemos por "comunicación" contribuirá a que la investigación en este campo sea más independiente y a que no se vea influenciada por otras disciplinas (Peters, 1986, p. 549). Nordestrend (1968, p. 208) señalaba, hace medio siglo, refiriéndose a la investigación en comunicación que había conocido en sus viajes a Estados Unidos, pero refiriéndose también a la investigación en comunicación que empezaba a ver en Europa, la existencia de un hipercientifismo. El investigador expresaba que veía mucho "crecimiento físico" y "muchos juegos con los que jugar", pero poco crecimiento intelectual y pocos problemas acerca de los que pensar, actividad ésta, pobremente representada en relación con los tipos de medida sofisticados que se estaban utilizando. Según el investigador, el campo se concentraba en usar correctamente los medios técnicos a expensas de perder nivel conceptual. Aquella realidad detectada por Nordestrend (1968) hace medio siglo, se ha acelerado frenéticamente y parece que fuera necesario, más que nunca, pararse a ubicar las perspectivas y los conceptos que han venido dando forma a la investigación en comunicación, para así conocer cuáles son los fundamentos del discurso actual y detectar posibles fundamentos alternativos a partir de los que el campo científico puede desarrollarse.

\section{Objetivos}

Es necesario construir y/o actualizar la dimensión intelectual de la investigación en comunicación para poder ir generando una perspectiva que tenga más incidencia en la sociedad. Para ir construyendo y dando forma a esa institucionalidad intelectual de la que, decíamos, se señala que carece la investigación en comunicación (Peters, 1986; Lacasa, 2017), parece importante compartir perspectivas e ideas acerca de al menos, tres asuntos fundamentales: 1) los diferentes significados que se le han venido dando a la comunicación y que han ido motivando la investigación que se realiza; 2) las diferentes perspectivas respecto del carácter científico o no de la comunicación y 3) los requisitos que se plantean como aquellos que pueden convertir a la investigación en comunicación en una disciplina.

A este objetivo, es decir, a construir, a dialogar y a generar alrededor de las tres cuestiones fundamentales planteadas, pretende contribuir el presente trabajo, situado en la confluencia de dos líneas de investigación: metainvestigación en comunicación e historia de la investigación en comunicación.

\section{Método de investigación y muestra}

Se ha aplicado un método de investigación utilizado en el contexto de un trabajo más amplio (Gómez-Diago, 2016) y que consiste, de manera fundamental en leer y en destilar artículos completos, un método que sigue los procesos definitorios ${ }^{2}$ de la práctica de la teoría fundamentada (Glaser y Strauss, 1967; Glaser, 1978; Strauss, 1987, en Charmaz, 2006, pp. 5-6), un método que, por tanto, evita el principal problema que, alerta Krippendorff (2017) implica el uso del análisis de

\footnotetext{
${ }^{2}$ De acuerdo con (Glaser y Strauss, 1967; Glaser, 1978; Strauss, 1987), los componentes definitorios de la práctica de la teoría fundamentada son:1) Participación simultánea en la recopilación y análisis de datos; 2) Construcción de códigos analíticos y categorías a partir de datos, no de hipótesis preconcebidas deducidas lógicamente; 3) Usar el método comparativo de forma constante, lo que implica hacer comparaciones durante cada etapa del análisis; 4) Avanzar en el desarrollo de la teoría durante cada paso de la recopilación de datos y análisis; 5) Redacción de notas para elaborar categorías, especificar sus propiedades, definir relaciones entre categorías e identificar brechas y 6) Muestreo dirigido a la construcción de teorías, no a la representatividad de la población.
} 
contenido y que radica en llevar lo que se quiere encontrar (categorías) a aquel lugar en el que se quiere encontrar.

El método utilizado está orientado a realizar una metainvestigación de las ideas que han venido motivando la investigación en comunicación. Este trabajo se centra en tres asuntos: 1) la concepción de la comunicación, es decir, los significados que puede tener la comunicación; 2) el carácter disciplinar de la comunicación y 3) los requisitos que tendría que tener el campo de la comunicación para convertirse en una ciencia.

A partir de un análisis de contenido cualitativo de tres volúmenes referentes en el campo de la comunicación y especializados en metainvestigación en comunicación, los volúmenes del Journal of Communication "Ferment in the Field" (1983) y "The Future of the Field. Between fragmentation and cohesion" (1993) y el volumen 1 de Rethinking Communication (1989), "Paradigm Issues", se han destilado algunas de las principales ideas que los investigadores autores de estas publicaciones aportan respecto de tres cuestiones fundamentales en la investigación en comunicación: 1) los significados que puede tener la comunicación; 2) el carácter disciplinar de la comunicación y 3) los requisitos que debe cumplir el campo de la investigación en comunicación para ser considerado como ciencia.

La elección de estos volúmenes viene motivada por ser referentes en este campo científico y por estar especializados en metainvestigación. El editor del "Ferment in the Field", Gerbner, solicitó a los autores que expresasen sus miradas acerca del estado de la Investigación en Comunicación en ese momento, específicamente, se les demandó a los investigadores que compartiesen sus opiniones acerca de la relación del investigador con la Ciencia, de la relación del investigador con la Sociedad, y con la Política, así como acerca de los objetivos que podría acometer la Investigación en Comunicación. El "Ferment in the field" está incluido en diferentes cronologías de la investigación en comunicación como la de Baran y Davis (2013) y es considerado por Nordestreng (2007, p. 212) como un volumen indispensable para la búsqueda de un "alma" que guíe la investigación en comunicación. Nordestreng $(2004$, p. 7$)$, tomando la idea de "ferment" de este volumen, ubica seis "ferments" en la investigación en comunicación ${ }^{3}$, definidos por la manera en la que la izquierda se relaciona con este campo científico. El investigador sitúa la publicación del "Ferment in the Field" en el segundo "ferment", momento en el que las perspectivas de izquierdas eran retadas por la creciente comercialización de los medios de comunicación y de la cultura.

El volumen del Journal of Communication "The Future of the Field, Between Fragmentation and Cohesion" (1993) fue editado por Levy y Gurevitch y la mayor parte de las contribuciones se centran en aspectos relacionados con aspectos sociales y políticos de la investigación en comunicación. Los editores habían sugerido a los autores que escribiesen acerca de algunos de los siguientes sugerentes enunciados: 1) la búsqueda de un paradigma en el campo se ha sustituido por una aceptación confortable del pluralismo teórico; 2) la investigación en comunicación es incapaz de influir en el periodismo y en las políticas públicas; 3) la investigación en comunicación carece de estatus disciplinar porque carece de centro de conocimiento; 4) la guerra fría acabó, pero las batallas ideológicas y metodológicas continúan fragmentado el campo; 5) el asunto de los efectos de los medios permanece en su estado perenne de caja negra y plantea cuestiones sin resolver.

\footnotetext{
${ }^{3}$ Nordestrend (2004) sitúa la aparición de cinco "ferment" en el campo de la investigación en función de cómo la izquierda se sitúa con el campo: en el primer ferment (1950s), la izquierda es invisible; en el segundo ferment (1960s), la izquierda está en la ofensiva; en el tercer ferment (1970), la izquierda se establece; en el cuatro ferment (1980), la izquierda es retada; en el quinto ferment (1990), la izquierda es cooptada y al sexto ferment (2000) lo denomina “¿Está volviendo la izquierda?".
} 
Finalmente, el volumen I del Rethinking Communication, titulado "Paradigm issues" (1989) fue editado por Dervin, Grossberg, O' Keefe, y Wartella y está configurado por cinco ensayos en los que Giddens, Hall, Krippendorff, Craig y Rosengren, desarrollan una mirada acerca de los aspectos fundamentales que el campo de la comunicación debe afrontar, identificando cada autor lo que considera que es el problema o los problemas que afectan a este campo científico, al mismo tiempo que proporcionan una imagen de cómo entienden que debe ser la investigación en comunicación. Además de los cinco ensayos, el volumen integra veinticinco comentarios que los editores solicitaron a investigadores de comunicación y de disciplinas relacionadas, representando un amplio rango de intereses, antecedentes, preferencias teóricas y políticas.

\section{4. $\quad$ Resultados}

Se organizan los resultados en tres categorías que son las que articulan el presente trabajo: 1) Significados de la comunicación; 2) Carácter disciplinar de la investigación en comunicación y 3) Criterios/procedimientos para convertir al campo de la comunicación en una disciplina.

\subsection{Significados de la comunicación}

Los investigadores autores de los artículos incluidos en los volúmenes estudiados entienden la comunicación, principalmente, de dos maneras: 1) como producto o resultado; y 2) como relación. Además, para definir la comunicación, los investigadores atienden principalmente e cuatro cuestiones: 1) a cómo toma forma la comunicación, 2) a los tipos de comunicación que pueden identificarse, 3) a las funciones que tiene y que puede tener la comunicación y 4) a la relación del campo de la comunicación con otras disciplinas.

Respecto de la consideración de la comunicación como producto o resultado, Balle y Cappe de Baillon (1983) definen a la comunicación social identificando tres posibles tipos de comunicación ${ }^{4}$ : 1) comunicación interpersonal, guiada por las costumbres; 2) comunicación institucional, que determina y que es determinada por el sistema social, por el sistema económico y por el sistema político; y 3) comunicación de medios, situada entre la comunicación interpersonal y la comunicación institucional.

El autor de la Teoría del Cultivo, Gerbner (1983) plantea la necesidad de centrar la investigación en la función de los mensajes. El investigador considera que el estudio de la comunicación gira alrededor de la producción, de la naturaleza y de la función de los mensajes en la vida y en la sociedad. En este sentido, el storytelling o la capacidad humana y ahora también de los programas informáticos para contar historias ${ }^{5}$, permite, de acuerdo con Gerbner, que la especie humana evolucione, y de ahí su importancia.

\footnotetext{
${ }^{4}$ La diferenciación de Balle y Cappe de Baillon (1983) se sigue utilizando cuarenta años después, y se le podría incorporar, por ejemplo la "autocomunicación de masas" (Castells, 2009), que se refiere a un tipo de comunicación que ni es interpersonal, ni es la comunicación que desarrollan los medios de comunicación, y tampoco es comunicación institucional.

${ }^{5}$ La capacidad para contar historias se ha visto aumentada por el desarrollo de las nuevas tecnologías, especialmente de Internet. Algoritmos como el programa de generación de lenguaje natural de Narrative Science pueden escribir historias originales al extraer contenido de grandes fuentes de datos. El storytelling que se realiza en el entorno virtual se estudia desde diferentes perspectivas, pero se hace necesario indagar, por ejemplo, en aspectos como la influencia que están ejerciendo los medios de comunicación tradicionales en lo que comentan los usuarios en unas redes sociales a las que esos medios de comunicación llevan las mismas temáticas que tratan en aquellos medios, involucrando además a los usuarios que se convierten en creadores y en difusores de esos contenidos.
} 
En cuanto a las propuestas que entienden a la comunicación como relación, Steeves (1993) subraya que la comunicación no es sólo la transmisión de mensajes de $\mathrm{A}$ a $\mathrm{B}$, sino que la comunicación engloba también el significado compartido y cambiante que se crea a través de relaciones de diferentes tipos, dentro de contextos económicos, políticos y culturales diferentes. Condit (1989) entiende la comunicación como algo que se diseña, haciendo referencia a su carácter generador y dinámico y subrayando que la comunicación es un proceso que construye sus distintivas y cambiantes posibilidades a través de la relación que se produce entre sus componentes. Por su parte, Dervin (1993) plantea la comunicación como el lugar donde lo micro se convierte en lo macro, donde se encuentran la estructura y el agente, el objeto y el individuo y la hegemonía y la resistencia. Esta propuesta de Dervin sitúa a la comunicación como eje a partir del cual se estructuran las relaciones. Su perspectiva ubica a la comunicación como puente entre esa estructura y los agentes, entendiendo la comunicación de forma similar a la manera en la que la entiende Habermas cuando sitúa a la comunicación como puente entre los sistemas y los "mundos de vida" (Gómez-Diago, 2019, p. 5).

Braman (1993) define a la comunicación como la manera en la que los elementos de un sistema participan en él creativamente, dando forma a ese sistema y a sus interacciones con otros sistemas. Desde una perspectiva que también incide en el carácter generador de la comunicación, Krippendorff (1989) entiende a la comunicación como la construcción interactiva de realidades que incluyen a los comunicadores que forman parte de ellas y que han creado y crean objetos durables como son el lenguaje, la tecnología y las instituciones sociales. La capacidad constructora de la comunicación que subraya Krippendorff es una de sus dimensiones fundamentales y es uno de los aspectos que hacen que sea tan necesario desarrollar una perspectiva capaz de abordar el estudio de de los diferentes tipos de relaciones que generan y que pueden generar los elementos y los actores que dan forma a diferentes relaciones comunicativas.

Finalmente, Schramm (1983) subraya la necesidad de considerar la comunicación como una transacción en la que dos partes (emisor y receptor) son activas. El investigador entiende la comunicación como una relación construida alrededor del intercambio de información y expresa que la comunicación siempre es parte de algo, que representa la relación no solo entre individuos, sino entre relaciones, siendo la red que une a la sociedad y siendo inclusiva en lugar de exclusiva.

A continuación, se incluye una tabla en la se sitúan los principales significados de comunicación recogidos de los volúmenes estudiados. 
RLCS, Revista Latina de Comunicación Social, 77, 393-412

[Investigación] DOI: 10.4185/RLCS-2020-1464 | ISSN 1138-5820 | Año 2020

Tabla 1. Principales significados de comunicación. Comunicación como producto/comunicación como relación.

\begin{tabular}{|c|c|}
\hline \multicolumn{2}{|c|}{ Significados de la comunicación } \\
\hline \multicolumn{2}{|c|}{ Comunicación como producto o resultado } \\
\hline $\begin{array}{l}\text { Balle y Cappe } \\
\text { de Baillon (1983) }\end{array}$ & $\begin{array}{l}\text { La comunicación social abarca desde la comunicación interpersonal hasta la } \\
\text { comunicación institucional, situándose entre ambas la comunicación de medios }\end{array}$ \\
\hline Gerbner (1983) & Lo fundamental es la capacidad de contar \\
\hline \multicolumn{2}{|c|}{ Comunicación como relación } \\
\hline Steeves (1993) & $\begin{array}{l}\text { Significado compartido y cambiante que se crea a través de relaciones de } \\
\text { diferentes tipos y de diferentes niveles, dentro de contextos económicos, políticos } \\
\text { y culturales particulares }\end{array}$ \\
\hline Condit (1989) & $\begin{array}{l}\text { Proceso que construye sus posibilidades a través de la relación entre sus } \\
\text { componentes }\end{array}$ \\
\hline Dervin (1993) & $\begin{array}{l}\text { La comunicación es donde lo micro se convierte en lo macro, lo macro en lo } \\
\text { micro }\end{array}$ \\
\hline Braman (1993) & $\begin{array}{l}\text { Manera en la que los elementos de un sistema participan creativamente dando } \\
\text { forma a ese sistema y a sus interacciones con otros sistemas }\end{array}$ \\
\hline $\begin{array}{l}\text { Krippendorff } \\
(1989)\end{array}$ & $\begin{array}{l}\text { Construcción interactiva de realidades que incluyen a los comunicadores que } \\
\text { forman parte de ellas y que han creado y crean objetos durables como son el } \\
\text { lenguaje, la tecnología y las instituciones sociales }\end{array}$ \\
\hline $\begin{array}{l}\text { Schramm } \\
(1983)\end{array}$ & $\begin{array}{l}\text { Relación construida en el intercambio de información mediante una transacción } \\
\text { en la que las dos partes (emisor y receptor) son activas }\end{array}$ \\
\hline
\end{tabular}

Fuente: Gómez-Diago, G. (2020).

\subsection{Carácter disciplinar de la investigación en comunicación}

Hall (1989) plantea que la comunicación no es una disciplina sino una teoría regional inextricablemente vinculada con las teorías sociales y contextualiza a la comunicación dentro de una Teoría Social amplia. El investigador considera que la comunicación es una teoría regional ligada al éxito y a la efectividad o a la inefectividad teórica de las teorías generales sociales en su conjunto porque es en este contexto en el que se tiene que teorizar el papel de la comunicación en el mundo social moderno. De acuerdo con Newcomb (1993) la comunicación no es una disciplina porque carece de un "centro del conocimiento", en el sentido de que se hacen investigaciones y acciones formativas acerca de aspectos muy reducidos de la realidad, generando especializaciones que apenas tienen desarrollo adentro de ellas mismas. Desde una perspectiva diferente, Craig $(1989,1993)$ y Bormann (1989) plantean la comunicación como una disciplina práctica. Craig (1989, 1993) considera que la disciplina de la comunicación es una disciplina configurada a partir de un tipo de teoría crítica orientada a la evaluación de prácticas comunicativas más que a proporcionar explicaciones causales o interpretaciones históricas y como metodología más apropiada para realizar la evaluación de prácticas comunicativas, el investigador propone utilizar metodologías empíricas y transformarlas a través de la incorporación de metodologías hermenéuticas. En una línea similar, Bormann (1989) sitúa a la comunicación como una disciplina práctica y a sus teorías especiales como una de las mayores fortalezas de la disciplina de la comunicación pues, según el investigador, mucho de lo que se hace tiene que ver con el estudio y la aplicación de teorías especiales a las necesidades diarias de comunicación de nuestras sociedades.

Pese a que investigadores como Herbst (2008) destacan que, para que haya interdisciplinariedad, debe haber antes disciplina, varios autores proponen entender el campo de la comunicación como 
una interdisciplina. En este sentido, Joch (1989) argumenta la importancia de considerar la comunicación como una interdisciplina para integrar perspectivas diferentes acerca de los procesos de comunicación social como las perspectivas de la Sociología y de la Speech communication, al mismo tiempo que subraya el hecho de que el campo de la comunicación alberga diferentes tradiciones. En la misma línea, Newcomb (1993), Pfau (2008) y Rowland (1993) consideran el campo de la comunicación como un campo interdisciplinar y entienden que es necesario que la investigación en comunicación parta del conocimiento de diversas epistemologías.

Considerando que el campo de la comunicación puede aportar una perspectiva diferenciadora, Beniger (1993) propone entender la comunicación como un medio para otra finalidad; propone entender la investigación en comunicación como un método para integrar modelos y datos de muchas disciplinas. En este sentido, el investigador expresa que el campo de la comunicación debe aspirar a convertirse en lo que Compte deseó para la Sociología, es decir, ser la reina de las Ciencias Sociales y conductuales, pues este campo cumple una de las condiciones necesarias, que es el hecho de que su objeto de estudio; la comunicación y la información, juegan un papel fundamental en las teorías centrales y en los modelos de todas las disciplinas relevantes.

Lang y Lang (1993), en una línea similar a la de Beniger, consideran que la comunicación es una perspectiva que puede arrojar luz en temas compartidos con otras disciplinas, y entienden la comunicación como una ciencia gerencial orientada a explicar un conjunto de problemas específicos. Jensen (1993) subraya el carácter social de la ciencia al expresar que la investigación en comunicación, de igual manera que el campo científico de la comunicación, es un mundo que hemos creado, algo que hacemos en común y que es constantemente redefinido por las prácticas sociales, culturales e interpretativas en las que nos involucramos. Por último, Halloran (1983) entiende a la comunicación como una ciencia multidisciplinar que necesita de la colaboración de otras ciencias.

A continuación, se incluye una tabla en la que se sitúan algunas perspectivas respecto del carácter disciplinar de la investigación en comunicación.

Tabla 2. Carácter disciplinar de la investigación en comunicación.

\begin{tabular}{|c|c|}
\hline \multicolumn{2}{|c|}{ Carácter disciplinar de la comunicación } \\
\hline Krippendorff (1989) & Construcción interactiva de realidades \\
\hline Beniger (1993) & Método para integrar modelos y datos de muchas disciplinas \\
\hline Lang y Lang (1993) & $\begin{array}{l}\text { Ciencia gerencial orientada a explicar un conjunto de problemas } \\
\text { específicos, perspectiva que puede arrojar luz en temas compartidos } \\
\text { con una o más disciplinas }\end{array}$ \\
\hline Bormann (1989) & Disciplina práctica \\
\hline Craig (1993) & $\begin{array}{l}\text { Disciplina práctica en la que la investigación crítica, interpretativa y } \\
\text { empírica, así como la reflexión filosófica y el trabajo aplicado, tienen } \\
\text { funciones esenciales }\end{array}$ \\
\hline Hall (1989) & $\begin{array}{l}\text { Teoría regional inextricablemente ligada a las teorías sociales, a su } \\
\text { efectividad o a su inefectividad }\end{array}$ \\
\hline $\begin{array}{l}\text { Joch Robinson (1989); Pfau } \\
\text { (2008) y Rowland (1993) }\end{array}$ & Interdisciplina \\
\hline Halloran (1983) & $\begin{array}{l}\text { Ciencia multidisciplinar, que necesita de la colaboración de otras } \\
\text { ciencias. }\end{array}$ \\
\hline
\end{tabular}

Fuente: Gómez-Diago, G. (2020). 
Algunos investigadores identifican algún aspecto diferencial en la investigación en comunicación y la sitúan como una ciencia para integrar datos de otras disciplinas, otros autores consideran a la comunicación como una "disciplina práctica", y se propone también a la comunicación como una Teoría social y como interdisciplina o ciencia interdisciplinar.

Una vez identificados algunos significados propuestos para la comunicación e identificadas diferentes perspectivas en lo que se refiere al carácter disciplinar de la investigación en comunicación, este trabajo se centra en algunos de los criterios aportados por los autores de los volúmenes analizados respecto de los requisitos que debería cumplir el campo de la comunicación para convertirse en una disciplina científica. Estos requisitos planteados por los investigadores como necesarios para que el campo de la comunicación se convierta en disciplina hacen referencia a una dimensión epistemológica, a una dimensión metodológica y a una dimensión institucional.

\subsection{Criterios/procedimientos para convertir el campo de la comunicación en una disciplina}

Pese a que O'Keefe (1993) considera que hay poca justificación para continuar buscando un consenso teórico unificado, subrayando que es más positivo para el campo de la comunicación promover tolerancia teórica y metodológica que buscar una cohesión disciplinar, y a pesar de que Miller (1989) expresa que es posible que lo universal nunca pueda ser tratado con éxito por los estudiosos de la comunicación, ya que el proceso de comunicación humana puede conceptualizarse y modelarse de muchas maneras, dependiendo de los objetivos del investigador y provocando que lo que constituye una explicación satisfactoria y una comprensión suficiente dependa de las funciones de una línea de investigación específica; diferentes autores de los volúmenes estudiados proponen criterios que debe cumplir la investigación en comunicación para ser considerada una disciplina científica. Dichos criterios hacen referencia a tres dimensiones del campo científico de la comunicación: una dimensión epistemológica, una dimensión institucional y una dimensión metodológica.

Desde una dimensión epistemológica, investigadores como Servaes (1989) y Katz (1983) subrayan que la comunicación, para desarrollarse como disciplina, debe incorporar otras disciplinas. En este sentido, Servaes (1989) plantea la necesidad de integrar perspectivas múltiples e interdisciplinarias, que presten atención a las miradas dominantes y Katz (1983), en la misma línea de pensamiento, destaca la necesidad de practicar una multidisciplinariedad en la que la investigación en comunicación incorpore las perspectivas de otras ciencias.

Lang y Lang (1993) subrayan la necesidad de que el campo de la comunicación tenga una perspectiva propia y expresan que para que la comunicación sea una disciplina, debe considerarse como algo más que un vehículo, que debe tener una ontología, es decir, una manera particular de mirar. Schramm (1986) plantea que la comunicación es una disciplina en el sentido de que ha logrado atraer a miles de investigadores que se reúnen, publican juntos, tienen puestos respetables en la academia y fuera de ella, y que enseñan un cuerpo de conocimiento bastante común, compartido; pero el investigador subraya la carencia de un cuerpo de teoría interrelacionado a partir del que los investigadores de la disciplina puedan construir y unificar su pensamiento. En este sentido, Carter (1989) señala el valor fundamental de la teoría para poder construir una disciplina de la comunicación. El investigador considera que para generar una disciplina es necesario producir un tipo de teoría que permita realizar formulaciones sobre las que construir, inventar e interpretar la práctica científica. En esta línea, Jakubowicz (1989) sitúa la responsabilidad de convertir el campo en una disciplina en los investigadores, que deben fusionar áreas y metodologías. 
Gerbner (1983) considera que para que el campo se convierta en disciplina, debe tener un dominio intelectual, un cuerpo de teorías y de perspectivas que sitúen su objeto de estudio. Desde una dimensión institucional, Gerbner (1983) plantea la necesidad de que haya organizaciones y también volúmenes que "alimenten" a sus integrantes.

Desde una perspectiva metodológica, Shepherd (1993) señala la necesidad de que el campo de la comunicación investigue las maneras en las que manifestaciones particulares de la existencia (individuos, sociedades) están comunicacionalmente (communicationally) construidas y Rogers y Chaffee (1983) proponen abandonar la perspectiva que entiende a la comunicación como algo lineal y que se centra en sus efectos para pasar a generar modelos de convergencia que permitan abordar los cambios introducidos por las nuevas tecnologías de la comunicación.

Daryl Slack y Allor (1983) proponen que, para que el campo de la comunicación proporcione una perspectiva de análisis diferenciada, los medios de comunicación y los procesos de la comunicación de masas deben estudiarse en relación con otras instituciones y procesos sociales como son el estado, la familia y la organización económica ${ }^{6}$.

Siguiendo con los criterios que, desde una perspectiva metodológica, necesitaría cumplir la investigación en comunicación para convertirse en un campo científico, Mancini (1993) hace referencia a la necesidad de vincular el campo de la comunicación con la sociedad, subrayando que para que la investigación en comunicación sea una disciplina, debe tener cuenta a la política y a la sociedad.

Davis y Jasinsky (1993) demandan la necesidad de que la investigación en comunicación influya en las instituciones, obligándoles a responder a las necesidades de los ciudadanos. La investigación en comunicación debe, según los investigadores, sugerir maneras de transformar las instituciones existentes, maneras de identificar y de promover el desarrollo de prácticas de comunicación innovadoras que sirvan para estructurar nuevos rituales, nuevos roles y nuevas instituciones, pues los investigadores consideran que la investigación busca una reforma microscópica cuando es necesario un cambio sustancial. En este sentido, Davis y Jasinsky (1993) expresan que hay abundante investigación orientada a que las instituciones y las personas mejoren en las maneras de comunicarse y que si bien esto ayudará a las personas a aceptar a las instituciones y a lidiar con un mundo cada vez más fragmentado, no va a aumentar el conocimiento acerca de su lugar en el mundo ni les va a motivar a buscar soluciones creativas e innovadoras a sus problemas. David y Jasinsky subrayan la necesidad de que la investigación en comunicación contribuya a generar una perspectiva a orientada a analizar la producción y la negociación de significados dentro de las comunidades, una investigación orientada al cambio social.

Por último, Rosengren (1993) destaca que para que el campo aporte una perspectiva diferenciada, debe plantearse una interacción entre la teoría sustantiva, los modelos formales y los datos empíricos, interacción, que, según el investigador a menudo falta en las tradiciones emergentes de la investigación en comunicación.

A continuación, se sitúan en una tabla, algunos de los criterios/procedimientos que plantean los investigadores autores de los volúmenes analizados para que el campo de la comunicación se convierta en una disciplina.

\footnotetext{
${ }^{6}$ La propuesta de estos investigadores cobra relevancia en un contexto en el que la mayor parte de la investigación realizada en las últimas décadas estudia los contenidos de los medios (Martínez Nicolás y Saperas, 2011, 2016; Anderson y Middleton, 2015); normalmente mediante un análisis de contenido cuantitativo que, expresábamos al principio del artículo, Krippendorff (2017) aconseja abandonar, principalmente, por devolver aquello que se busca.
} 
RLCS, Revista Latina de Comunicación Social, 77, 393-412

[Investigación] DOI: 10.4185/RLCS-2020-1464 | ISSN 1138-5820 | Año 2020

Tabla 3. Criterios/procedimientos para convertir el campo de la comunicación en una disciplina.

\begin{tabular}{|c|c|}
\hline \multicolumn{2}{|c|}{$\begin{array}{l}\text { Criterios/procedimientos para convertir al campo de la investigación en comunicación en una } \\
\text { disciplina }\end{array}$} \\
\hline Servaes (1989) & $\begin{array}{l}\text { Perspectivas múltiples e interdisciplinarias, que presten atención a las } \\
\text { miradas dominantes y a las alternativas }\end{array}$ \\
\hline Schramm (1983) & Conformar una ciencia del hombre con otras disciplinas \\
\hline Katz (1983) & Multidisciplinariedad \\
\hline Gerbner (1983) & $\begin{array}{l}\text { Dominio intelectual, teorías y perspectivas que sitúen su objeto de estudio, } \\
\text { así como existencia de organizaciones profesionales y de volúmenes que } \\
\text { informen, socialicen y alimenten a sus miembros }\end{array}$ \\
\hline Carter & $\begin{array}{l}\text { Teoría que permita realizar formulación sobre la que construir, inventar e } \\
\text { interpretar la práctica }\end{array}$ \\
\hline Jakubowicz (1989) & Fusionar áreas y metodologías en un conjunto diferenciado coherente \\
\hline Shepherd (1989) & $\begin{array}{l}\text { Investigar las maneras en las que manifestaciones particulares de la } \\
\text { existencia (individuos, sociedades) están comunicacionalmente } \\
\text { (communicationally) construidos }\end{array}$ \\
\hline $\begin{array}{l}\text { Everett \& Chaffee } \\
(1983)\end{array}$ & $\begin{array}{l}\text { Abandonar los efectos lineales para cambiar hacia modelos de convergencia } \\
\text { de comunicación que acomoden la interactividad de la mayoría de las } \\
\text { tecnologías de la comunicación }\end{array}$ \\
\hline $\begin{array}{l}\text { Daryl Slack y Allor } \\
\text { (1983) }\end{array}$ & $\begin{array}{l}\text { Entender los procesos de la comunicación de masas en relación con otras } \\
\text { instituciones sociales y otros procesos sociales como son el estado, la } \\
\text { familia y la organización económica }\end{array}$ \\
\hline Mancini (1983) & Tener en cuenta la comunicación política y la sociedad \\
\hline $\begin{array}{l}\text { Davis \& Jasinsky } \\
\text { (1993) }\end{array}$ & Encaminar la investigación en comunicación a influir en las instituciones \\
\hline Rosengren (1989) & Vincular la teoría sustantiva, los modelos formales y los datos empíricos \\
\hline
\end{tabular}

Fuente: Gómez-Diago, G. (2020).

Al mismo tiempo que a algunos investigadores les preocupa identificar criterios para que la investigación en comunicación alcance un estatus de disciplina, investigadores como Hamelink (1983) y Thayer (1983) plantean la necesidad de que la investigación en comunicación se libere de los métodos de investigación considerados como científicos para integrar otros modos de análisis y de trabajo más vinculados a la creatividad. En este sentido, Hamelink subrayaba en el "Ferment in the Field" la necesidad de liberar a la investigación de la forma usual de generar conocimiento puesto que, según el investigador, la dimensión científica no ha demostrado su superioridad sobre otras dimensiones más intuitivas de la vida humana.

Para buscar la emancipación de la investigación en comunicación, Hamelink (1983) propone utilizar más arte que ciencia. De forma similar, Thayer (1983) explica que el científico intenta emular el supuesto método de la ciencia de laboratorio, siendo esto, de acuerdo con el investigador, un error dado que la ciencia de laboratorio no predice nada que no controle, que no esté completamente determinado. De acuerdo con Thayer es imposible estudiar sistemas abiertos con métodos solo apropiados para sistemas cerrados y esta miopía explica, según el investigador, el carácter irrelevante de la mayor parte de la investigación social en los Estados Unidos hasta la fecha.

\section{Discusión y Conclusiones}

Este trabajo ha traído perspectivas expresadas en tres volúmenes fundamentales en metainvestigación en comunicación respecto de tres aspectos: 1) significados de la comunicación, 2) carácter disciplinar 
del campo y 3) posibles criterios para convertir al campo de la comunicación en disciplina. En lo que se refiere a las maneras de abordar la comunicación, los autores de los artículos estudiados entienden la comunicación como producto o como relación. Es esta última perspectiva, la que entiende a la comunicación como relación, la más adecuada para abordar la investigación en este campo científico, pues entender la comunicación como relación implica mirar más allá de la "comunicación", posibilitando analizar un determinado contexto desde una perspectiva comunicativa. Desde esta perspectiva se entiende la comunicación como origen de las relaciones y, por tanto, como generadora de determinadas instituciones, asociaciones o sociedades. Desarrollando esta perspectiva es que se ha venido planteando la idea de considerar a la comunicación como contexto para la interacción (Gómez-Diago, 2016, 2017.b, 2018, 2019), perspectiva que facilita abordar la comunicación como espacio y como variable dependiente. En este sentido, para superar la contradicción que encuentra Peters (2008, p. 143) en querer sustentar la especificidad de la investigación en comunicación solamente en el hecho de que se preocupa por algo que despierta tanto interés como la comunicación, el campo científico de la comunicación puede ayudar a pensar comunicacionalmente (Deetz, 2010).

Los criterios planteados en los tres volúmenes analizados para que el campo de la comunicación se convierta en una ciencia, hacen referencia a tres dimensiones: una dimensión institucional, una dimensión epistemológica y una dimensión metodológica. Desde una dimensión epistemológica se subraya la carencia que tiene el campo de la comunicación de trabajos teóricos y de teorías. Esto sigue ocurriendo en la actualidad y queda evidenciado en estudios como el de Anderson y Middleton (2015) en el que se concluye que en la investigación en comunicación, y a pesar de los cambios que ha motivado la extensión en el uso de las nuevas tecnologías, se siguen utilizando las mismas teorías que hace cincuenta años, es decir, mientras las tecnologías están sufriendo continuos cambios, las perspectivas para investigar acerca de estos cambios permanecen en un estado de inercia (Wang, 2011, p. 1458). Quizá este estado de inercia se debe a la escasez de artículos teóricos (Martínez Nicolás y Saperas, 2011, 2016; Neuman y Guggenheimm, 2011; Bryant y Miron, 2004) y quizá la carestía de producción teórica se produce debido a la necesidad que se impone de publicar (Haniztsch, 2015, p. 351), necesidad que parece motivar el uso de técnicas de investigación fundamentalmente cuantitativas (Martínez Nicolás y Saperas, 2011; 2016), técnicas que ya eran las más usadas hace tres lustros (Cooper et al., 1994).

Finalmente, desde una dimensión metodológica, se sugiere que el campo de la comunicación investigue las maneras en las que la existencia de los individuos y de las sociedades están comunicacionalmente construidos, se recomienda abandonar el estudio de los efectos lineales para incorporar el uso de modelos de convergencia que permitan estudiar la interactividad de las tecnologías de la comunicación, se propone entender los procesos de la comunicación de masas en relación con otras instituciones sociales como son el estado, la familia y la organización económica; se subraya la necesidad de tener en cuenta la comunicación política y la sociedad y también se destaca la necesidad de que la investigación en comunicación busque influir en las instituciones.

Aún teniendo en cuenta que un método es insuficiente para hacer buena investigación, y que la aplicación de métodos de manera mecanicista lleva a resultados irrelevantes, siendo en ese caso más útil una mirada y un oído atento que cualquier otro procedimiento metodológico (Charmaz \& Mitchell, 1996, p. 15); es fundamental desarrollar propuestas metodológicas para la investigación en comunicación pues van a ser vías que permitan generar nuevas teorías que consoliden a este campo científico que puede ser de ayuda en una sociedad cada vez más desigual. En este sentido de la necesidad de generar metodologías de investigación que proporcionen resultados diferentes, Hamelink (2018, p. 3) sigue planteando que, además de al conocimiento científico, se tome en serio al conocimiento experimental y tácito de fuentes no científicas y Martínez Nicolás (2019; pp. 13-14), 
en la Lección inaugural del curso académico 2019-2020 en la Universidad Rey Juan Carlos, anima a los investigadores sociales a "trabajar junto con la sociedad haciéndoles copartícipes del proceso de la investigación, pero ahora ya de una forma generalizada y sistemática, explorando a conciencia todas las posibilidades de esa intervención social en el planeamiento, la ejecución y la explotación de los resultados de nuestros proyectos de investigación".

Incluir a los destinatarios de la investigación en su diseño y en su transcurso, dándoles voz, puede generar nuevas formas de investigar que vayan consolidando intelectualmente y socialmente al campo científico de la comunicación. En esta línea de interés, un asunto relevante para ser abordado en futuros trabajos, es determinar quiénes han venido siendo, quiénes son y quiénes pueden ser los destinatarios de la investigación en comunicación. Esta cuestión está muy vinculada con cuáles han venido siendo, con cuáles son y con cuáles pueden ser los objetivos de la investigación en comunicación, y son cuestiones que obligan a generar una discusión intelectual necesaria que posibilite ir redefiniendo y/o ampliando el foco y la dimensión de un campo científico, el de la comunicación, que puede ser de utilidad a la sociedad.

\section{Referencias}

Anderson, J. A., \& Middleton, M. K. (2015). Epistemological Movements in Communication. An Analysis of Empirical and Rhetorical Critical Scholarship. In Pat J. Gehrke y William M. Keith (eds): A Century of Communication Studies: The Unfinished Conversation. Routledge.

Balle, F., \& Cappe de Baillon, I. (1983). Mass Media Research in France: An Emerging Discipline. Journal of Communication, 33(3), 146-156. https://doi.org/10.1111/j.1460-2466.1983.tb02416.x

Baran, S. J., \& Davis, D. K. (2012). Mass Communication Theory. Foundations. Ferment, and Future. Sixth edition. Wadsworth Cengage Learning.

Beniger, J. R. (1993). Communication-Embrace the Subject, not the Field. Journal of Communication, 43(3), 18-25. https://doi.org/10.1111/j.1460-2466.1993.tb01272.x

Bormann, E. (1989). On Communication as a Practical Discipline. Rethinking Communication, "Paradigm Issues". (Vol. 1, 135-138). Sage.

Braman, S. (1993). Harmonization of Systems: The Third Stage of the Information Society. Journal of Communication, 4(3), 133-140. https://doi.org/10.1111/j.1460-2466.1993.tb01285.x

Bryant, J., \& Miron, D. (2004). Theory and research in mass communication. Journal of Communication, 54(4), 662-704. https://doi.org/10.1111/j.1460-2466.2004.tb02650.x

Cáceres, M. D. y Caffarel, C. (1992). La Investigación sobre Comunicación en España. Un Balance Cualitativo. Telos, (32), 109-124.

http://www.quadernsdigitals.net/datos/hemeroteca/r 32/nr 447/a 6136/6136.pdf

Carter, R. F. (1989). On Paradigmatic Productivity. Rethinking Communication. "Paradigm Issues". (Vol. 1, 145-147). Sage.

Castells, M. (2009). Comunicación y Poder. Alianza Editorial. 
RLCS, Revista Latina de Comunicación Social, 77, 393-412

[Investigación] DOI: 10.4185/RLCS-2020-1464 | ISSN 1138-5820 | Año 2020

Condit, C. (1989). Replacing Oxymora: Instituting Communication Studies, Rethinking communication, "Paradigm issues". (Vol. 1). Sage.

Cooper, R., Potter, W, \& Dupagne, M. (1994). A Status Report on Methods Used in Mass Communication Research. Journalism Educator, 48(4), 54-61.

https://doi.org/10.1177/107769589304800408

Corner, J. (1979). "Mass" in Communication Research. Journal of Communication, 29(1), $26-32$. https://doi.org/10.1111/j.1460-2466.1979.tb01679.x

Craig, R. T. (1993). Why Are There So Many Communication Theories? Journal of Communication, 43(3), 26-33. https://doi.org/10.1111/j.1460-2466.1993.tb01273.x

Craig, R. T. (1989). Communication as a Practical Discipline. Rethinking Communication. "Paradigm Issues". (Vol. 1, 97-122). Sage.

Charmaz, K. (2006). Constructing Grounded Theory: A Practical Guide Through Qualitative Analysis. Sage Publications.

Daryl Slack., J. y Allor, M. (1983). The Political and Epistemological Constituents of Critical Communication Research. Journal of Communication, 33(3), 208-218. https://doi.org/10.1111/j.1460-2466.1983.tb02421.x

Davis, D. K y Jasinski, J. (1993). Beyond the Culture Wars: An Agenda for Research on Communication and Culture. Journal of Communication, 43(3), 141-149. https://doi.org/10.1111/j.1460-2466.1993.tb01286.x

Danziger, K. (2003). Where history, theory, and philosophy meet: The biography of psychological objects. En D. B. Hill \& M. J. Kral (Eds.), SUNY series, alternatives in psychology. About psychology: Essays at the crossroads of history, theory, and philosophy (pp. 19-33). State University of New York Press.

Deetz, S. (2010). Politically Attentive Relational Constructionism (PARC) Making a difference in a Pluralistic Independent World. En Donald Carbaugh y Patrice Buzzanell (eds), Distinctive Qualities in Communication Research. Routledge.

Dervin, B. (1993). Verbing Communication: Mandate for Disciplinary Invention. Journal of Communication, 43(3), 45-54. https://doi.org/10.1111/j.1460-2466.1993.tb01275.x

Eurostat (20/04/2020). Unemployment rate. Anual data.

https://ec.europa.eu/eurostat/databrowser/view/tipsun20/default/table?lang=en

Ewen, S. (1983). The Implications of Empiricism. Journal of Communication, 33(3), 219-225. https://doi.org/10.1111/j.1460-2466.1983.tb02422.x

Gerbner, G. (1983). The Importance of Being Critical-In One's Own Fashion. Journal of Communication, 33(3), 355-362. https://doi.org/10.1111/j.1460-2466.1983.tb02435.x

Gómez-Diago, G. (2019). A threefold approach for social change: uneven development, recognition and communication as context for interaction. In Jan Servaes (ed.), Handbook of Communication 
for Development and Social Change. (Section 4: Cluster of concepts that sets an important context for communication activities for development). Springer. https://doi.org/10.1007/978-981-107035-8, https://cutt.ly/Tub190E

Gómez-Diago, G. (2018). Paradigma para la Teoría Crítica en la investigación en comunicación: la comunicación como contexto para la interacción. VI Congreso Internacional de la AE-IC, “Comunicación y Conocimiento”. (pp. 37-51). Universidad de Salamanca. https://cutt.ly/CubGdYE

Gómez-Diago, G. (2017.a). Herramientas conceptuales para la investigación en comunicación en tres volúmenes principales de metainvestigación en comunicación. XV Congreso Internacional Ibercom. (pp. 136-152). Assibercom. https://shorturl.at/hotyZ

Gómez-Diago, G. (2017.b). El papel de la investigación en comunicación ante la participación ciudadana. Propuesta para investigar entendiendo la comunicación como contexto para la interacción. En Javier Herrero y Concha Mateos (eds.), Del verbo al bit. (2 ${ }^{\circ}$ Edición ampliada, pp. 1879-1899). Sociedad Latina de Comunicación Social. https://doi.org/10.4185/cac116edicion2 https://dialnet.unirioja.es/servlet/articulo?codigo $=6071964$

Gómez-Diago, G. (2016). Para investigar en comunicación. 400 ideas y una propuesta para actualizar el paradigma de la interacción (Tesis Doctoral. Premio Extraordinario de Doctorado). Universidad Rey Juan Carlos. Director: Manuel Martínez-Nicolás.

Gunkel, D. (junio, 19, 2015). Man Vs Machine. Northen Illinois University. NIU. http://www.niutoday.info/2015/06/19/man-vs-machine/

Habermas, J. (1987). The theory of communicative action. Volume 2. Lifeworld and system: a critique of functionalist reason. Beacon Press.

Hackett, R. A. (1984). Decline of a paradigm? Bias and objectivity in news media studies. CSMC. Critical Studies in Mass Communication, 1(3), 229-259. https://doi.org/10.1080/15295038409360036

Hall, S. (1989). Ideology and Communication Theory. Rethinking Communication. "Paradigm Issues". (Vol. 1, 40-52). Sage.

Halloran, J. (1983). A Case for Critical Eclecticism, Journal of Communication, 33(3), 270-278. https://doi.org/10.1111/j.1460-2466.1983.tb02428.x.

Hamelink, C. J. (2018). Communication Research: Resignation or Optimism? Javnost - The Public, 25(1-2), 218-225. https://doi.org/10.1111/j.1460-2466.1993.tb01272.x

Hamelink, C. J. (1983). Emancipation or Domestication: Toward a Utopian Science of Communication, Journal of Communication, 33(3), 74-79. https://doi.org/10.1111/j.14602466.1983.tb02408.x

Hanitzsch, T. (2015). Celebrating 25 Years of Communication Theory: Growing Diversity Under Heavy Strain, Communication Theory, 25(4), 349-355. https://doi.org/10.1111/comt.12079 
RLCS, Revista Latina de Comunicación Social, 77, 393-412

[Investigación] DOI: 10.4185/RLCS-2020-1464 | ISSN 1138-5820 | Año 2020

Herbst, S. (2008). Disciplines, Intersections, and the Future of Communication Research. Journal of Communication, 58(4), 603-614. https://doi.org/10.1111/j.1460-2466.2008.00402.x

Jakubowicz, K. (1989). Paradigm Dialogues: Navel-Gazing or Bridge-Building. Rethinking Communication. "Paradigm Issues". (Vol. 1. 179-183). Sage.

Jensen, J. (1993). The Consequences of Vocabularies. Journal of Communication, 43(3), 67-74. https://doi.org/10.1111/j.1460-2466.1993.tb01277.x

Joch Robinson, G. (1989). Communication Paradigm Dialogues: Their Place in the History of Science Debate. Rethinking Communication. "Paradigm Issues”. (Vol. 1, 205-297). Sage.

Katz, E. (1983). The Return of the Humanities and Sociology. Journal of Communication, 33(3), 5152. https://doi.org/10.1111/j.1460-2466.1983.tb02405.x

Krippendorff, K. (2017). Three concepts to retire. Annals of the International Communication Association, 41(1). https://doi.org/10.1080/23808985.2017.1291281

Krippendorff, K. (1989). On the Ethics of Constructing Communication. Rethinking Communication. "Paradigm Issues". (Vol. 1, 66-95). Sage.

Lacasa-Mas, I. (2017). Die spanische Kommunikationswissenschaft auf dem Weg zu internationaler Anerkennung. Ein Abriss der Fachgeschichte, Kommunikationswissenschaft im internationalen Vergleich. Transnationale Perspektiven. (pp. 185-210). Springer.

Lang, K. y Lang, E. G. (1993). Perspectives on Communication. Journal of Communication, 43(3), 92-99. https://doi.org/10.1111/j.1460-2466.1993.tb01280.x

Mancini, P. (1993). The Legitimacy Gap: A Problem of Mass Media Research in Europe and the United States. Journal of Communication, 43(3), 100-109. https://doi.org/10.1111/j.14602466.1993.tb01281.x

Martínez-Nicolás, M. (2019)."¿Sirven para algo las ciencias sociales? Desafíos a la investigación social y responsabilidad de la comunidad científica". Lección inaugural del curso académico 2019-2020 de la Universidad Rey Juan Carlos (URJC). 17 de septiembre, 2019. Lección escrita: https://cutt.ly/RryJDRr. Vídeo de la lección: https://cutt.ly/lryLwVk (37-1h.20). Vídeo resumen de la lección: https://cutt.ly/yryLeEE (3.38).

Martínez-Nicolás, M. y Saperas, E. (2016). Objetos de estudio y orientación metodológica de la reciente investigación sobre comunicación en España (2008-2014). Revista Latina de Comunicación Social, (71), 1365-1384. https://doi.org/10.4185/RLCS-2016-1150 https://www.redalyc.org/articulo.oa?id=81943468070

Martínez-Nicolás, M. y Saperas, E. (2011). La investigación sobre Comunicación en España (19982007). Análisis de los artículos publicados en revistas científicas. Revista Latina de Comunicación Social, (66), 101-129. https://doi.org/10.4185/RLCS-66-2011-926-101-129

http://www.revistalatinacs.org/11/art/926_Vicalvaro/05 Nicolas.html

Mattelart, A. (1983). Technology, Culture, and Communication: Research and Policy Priorities in France. Journal of Communication, 33(3), 59-73. 
RLCS, Revista Latina de Comunicación Social, 77, 393-412

[Investigación] DOI: 10.4185/RLCS-2020-1464 | ISSN 1138-5820 | Año 2020

https://doi.org/10.1111/j.1460-2466.1983.tb02407.x

Miller, G. (1989). Taking Stock of a Discipline. Journal of Communication, 33(3), 3-41. https://doi.org/10.1111/j.1460-2466.1983.tb02403.x

Mills W. Ch. (1987). La Imaginación Sociológica. Fondo De Cultura Económica.

Neuman, W. y Guggenheim, L. (2011). The evolution of media effects theory: a six-stage model of cumulative research. Communication Theory, 21(2), 169-196. https://doi.org/10.1111/j.1468$\underline{2885.2011 .01381 . x}$

Newcomb, H. (1993). Target Practice: A Batesonian "Field" Guide for Communication Studies. Journal of Communication, 43(3), 127-132, https://doi.org/10.1111/j.1460-2466.1993.tb01284.x

Nordenstreng, K. (2007). Discipline or Field? Nordicom Review, Jubilee Issue 2007, 211-222. https://www.nordicom.gu.se/sites/default/files/kapitel-pdf/248_248_nordenstreng1.pdf

Nordenstreng, K. (2004). Ferment in the Field: Notes on the Evolution of Communication Studies ad its Disciplinary Nature. Javonost-The Public, 11(3), 5-17.

https://doi.org/10.1080/13183222.2004.11008856

Nordenstreng, K. (1968). Communication Research in the United States: a Critical Perspective. Gazette, 14(3), 207-216. https://doi.org/10.1177/001654926801400303

O'Keefe, B. (1993). Against Theory. Journal of Communication, 43(3), 75-82.

https://doi.org/10.1111/j.1460-2466.1993.tb01278.x

Peters J. D. (1986). Institutional sources of intellectual poverty in communication research. Communication Research. 13(4), 527-559. https://doi.org/10.1177/009365086013004002

Peters, J. D. (2008). Institutional Opportunities for Intellectual History in Commmunication Studies, 143-162. In David W. Park y Jefferson Pooley (eds.), The History of Media and Communication Research. Contested Memories. Peter Lang.

Pfau, M. (2008). Epistemological and Disciplinary Intersections. Journal of Communication, 58(4), 597-602. https://doi.org/10.1111/j.1460-2466.2008.00414

Rheinberger, H. J. (2011). Consistency from the perspective of an experimental systems approach to the sciences and their epistemic objects, Manuscrito, 34(1). https://doi.org/10.1590/S0100$\underline{60452011000100014}$

Ritzer, G. (1990). Metatheorizing in Sociology. Lexington Books Series on Social Theory.

Rogers, E. M., \& Chaffee, S. (1983). Communication as an Academic Discipline: A Dialogue. Journal of Communication, 33(3), 18-30. https://doi.org/10.1111/j.1460-2466.1983.tb02402.x

Rosengren, K. E. (1993). From Field to Frog Ponds, Journal of Communication, 43(3) 6-17. https://doi.org/10.1111/j.1460-2466.1993.tb01271.x

Rowland, Willard D. Jr (1993). The Traditions of Communication Research and Their Implications for Telecommunications Study. Journal of Communication, 43(3) 207-217. 
RLCS, Revista Latina de Comunicación Social, 77, 393-412

[Investigación] DOI: 10.4185/RLCS-2020-1464 | ISSN 1138-5820 | Año 2020

https://doi.org/10.1111/j.1460-2466.1993.tb01294.x

Schramm, W. (1983). The Unique Perspective of Communication: A Retrospective View. Journal of Communication, 33(6), 6-17. https://doi.org/10.1111/j.1460-2466.1983.tb02401.x

Servaes, J. (1989). Après le Deluge. En Rethinking Communication. "Paradigm Issues". (Vol. 1, 214-218). Sage.

Shepherd, G. J. (1993). Building a Discipline of Communication. Journal of Communication, 43(3), 83-91. https://doi.org/10.1111/j.1460-2466.1993.tb01279.x

Steeves, H. L. (1993). Creating Imagined Communities: Development Communication and the Challenge of Feminism. Journal of Communication, 43(3), 218-229.

https://doi.org/10.1111/j.1460-2466.1993.tb01295.x

Surlin, S. (1991). Rethinking Communication, vol.1. Paradigm Issues. Vol.2.Paradigm Exemplars. Canadian Journal of Communication, 16(3).

https://www.cjc-online.ca/index.php/journal/article/view/642/548

Thayer, L. (1983). On Doing Research and Explaining Things. Journal of Communication, 33(3), 8091. https://doi.org/10.1111/j.1460-2466.1983.tb02409.x

Wang, G. (2011). Plenary Paradigm Shift and the Centrality of Communication Discipline. International Journal Of Communication, (5), 9. https://ijoc.org/index.php/ijoc/article/view/1335

White, R. A. (1983). Mass Communication and Culture: Transition to a New Paradigm. Journal of Communication, 33(3), 279-301. https://doi.org/10.1111/j.1460-2466.1983.tb02429.x

\section{AUTORA:}

\section{Gloria Gómez-Diago}

Profesora ayudante doctora en la Universidad Rey Juan Carlos (URJC). Doctora en Ciencias de la Comunicación. Premio Extraordinario de Doctorado con la tesis doctoral "Para Investigar en comunicación. 400 ideas de las últimas tres décadas y una propuesta para actualizar el paradigma de la interacción". Líneas de investigación: Teorías y métodos para la investigación en comunicación, historia de la investigación en comunicación, metainvestigación en comunicación, formas de participación ciudadana y metodologías para el aprendizaje y la docencia. En estas líneas de investigación ha publicado más de treinta trabajos (artículos, capítulos, entradas en enciclopedias) y ha presentado más de cuarenta comunicaciones en congresos nacionales e Internacionales.

Índice H: 9.

Google Scholar: https://scholar.google.com/citations?user=fZezpPoAAAAJ\&hl=en

\section{Algunos de los últimos trabajos publicados}

GÓMEZ-DIAGO, G. (2019). A Threefold approach for enabling social change: Communication as Context for Interaction, Uneven Development, and Recognition ». En J. Servaes J. (ed.): Handbook of Communication for Development and Social Change, Singapore: Springer, pp.1-14. https://doi.org/10.1007/978-981-10-7035-8-7-1

GÓMEZ-DIAGO, G. (2019). Paradigm shift. En D. Merskin (ed.): International Encyclopedia of Mass Media and Society. The SAGE international encyclopedia of mass media and society, Vol. 
1. Thousand Oaks,, CA: SAGE Publications, Inc. pp. 1333-1334. doi: 10.4135/9781483375519.n509

GÓMEZ-DIAGO, G. (2019). Funcionalist theory. En D. Merskin (ed.): The SAGE international encyclopedia of mass media and society, Vol. 1. Thousand Oaks, CA: SAGE Publications, Inc. pp. 657-658. doi: 10.4135/9781483375519.n260

GÓMEZ-DIAGO, G. (2019). Metainvestigación en comunicación en España. Propuesta de una tipología

En F. Sierra y J. Alberich (eds.): Epistemología de la comunicación y cultura digital: retos emergentes. Granada: Universidad de Granada, pp. 271-284.

GÓMEZ-DIAGO, G. (2018). Paradigma para la Teoría Crítica en la Investigación en Comunicación: la comunicación como contexto para la interacción. VI Congreso Internacional de la AE-IC, "Comunicación y Conocimiento". Salamanca: Asociación española para la investigación en comunicación, pp. 37-51.

GÓMEZ-DIAGO, G. (2018). Propuesta socio-crítica para investigar las culturas periodísticas a partir de la revisión de Worlds of Journalism Study (2012-2015). Estudios sobre el Mensaje Periodístico, 24(1), pp.173-191. https://doi.org/10.5209/ESMP.59944

GÓMEZ-DIAGO, G. (2017). Indicadores para definir la participación ciudadana y/o política. Una propuesta: de lo personal a lo público. En José Candón Mena (ed.): Actas del III Congreso Internacional Move.net sobre Movimientos sociales y TIC, pp. 176-189.

GÓMEZ-DIAGO, G. (2016). The role of shared emotions in the construction of the Cyberculture. From cultural industries to cultural actions. The case of crowdfunding. En S. Y. Tettegah (ed.): Emotions, Technology and Social Media, pp. 49-62. London (UK), San Diego, CA (USA), Cambridge, MA (USA), Oxford (UK): Elsevier. https://doi.org/10.1016/B978-0-12-801857$6.00003-8$ 